\title{
Historia del sufijo tenencial del quechua: reconstrucción y nuevas hipótesis
}

\author{
Carlos Alberto Faucet Pareja \\ Universidad Nacional Mayor de San Marcos \\ carlos.faucet@unmsm.edu.pe
}

\begin{abstract}
Resumen
En este trabajo de linguiística histórica quechua se utiliza la metodología consolidada de la Linguiística histórica (método comparativo y método de reconstrucción interna) para, primero, evaluar el estado de la cuestión acerca de la historia del sufijo tenencial (-yuq) y, segundo, efectuar la reconstrucción de dicho sufijo. Por último, se extraen las consecuencias de la reconstrucción anterior ("-nyuq) y se propone una nueva hipótesis que intenta retroceder aún más en la historia.

Palabras claves: sufijo tenencial quechua -yuq, Linguiística histórica, método comparativo, reconstrucción interna

Abstract

In this paper of quechuan historical linguistics the accepted methodology of Historical Linguistics (Comparative Method and Internal Reconstruction) is used in order to assess the current proposals about the history of "tenencial" suffix (-yuq) and also proceed to its very reconstruction. Finally, the consequences of this reconstruction ("-nyuq) are taken on and a new hypothesis that attempts to go further back in history is advanced.
\end{abstract}

Keywords: Quechua suffix -yuq, Historical Linguistics, Comparative Method, Internal Reconstruction

Recibido: 2/3/2015 Aceptado: 25/4/2015 


\section{Introducción}

La linguiística (histórica) quechua comenzó a ser trabajada rigurosamente solo a partir de la segunda mitad del siglo XX, con las obras fundamentales de Parker (1963) y Torero (1964). Desde entonces, a nivel diacrónico, se ha avanzado con bastante seguridad respecto a lo fonológico, en menor medida en lo léxico y en lo morfosintáctico, y casi nada en lo semántico.

En cuanto a lo morfosintáctico, uno de los muchos elementos cuyo origen permanece oscuro es el muy productivo sufijo tenencial (-yuq, su forma más conocida). Las propuestas acerca de su historia tienen algún punto en común y muchos en desacuerdo, pero, sobre todo, han sido realizadas, en el mejor de los casos, pasando por alto algunos presupuestos de la Linguiística histórica (LH), cuando no han sido simples enunciaciones sin una argumentación que las respaldara.

En consideración de lo anterior, y siguiendo una idea de Moreno Cabrera1, en este trabajo empleo los presupuestos del método comparativo (MC), además del método de reconstrucción interna (MRI), para evaluar el estado de la cuestión acerca de la historia del sufijo tenencial y realizar, luego, mi propuesta de reconstrucción, la cual, aunque válida en sí misma, me servirá también, finalmente, para generar a partir de ella nuevas hipótesis que intentarán retroceder aun más en la historia del sufijo.

\section{Los métodos de la LH}

La LH trata del cambio linguiístico: cómo y por qué las lenguas cambian (Campbell 1999). Su nacimiento en el siglo XIX es el nacimiento de la Linguiística como disciplina científica (Coseriu 1981). Desde entonces, la LH ha consolidado dos instrumentos empíricos para reconstruir un estado de lengua a partir de la evidencia posterior: el MC y el MRI (Hoenigswald 1965). La diferencia entre ellos reside en que el MC trabaja con cognados, morfemas semejantes en forma y significado pertenecientes a lenguas emparentables, en tanto que el MRI trabaja dentro de una misma lengua, con alomorfos e irregularidades morfológicas (Campbell 1999). Ya que la reconstrucción se realiza a partir de la evidencia posterior, se sobreentiende que toda empresa reconstructiva de-

1 Moreno Cabrera (1997: 79): «los resultados de la aplicación correcta y adecuada del método [comparativo] no tienen el status de especulaciones gratuitas basadas en los prejuicios o en la fantasía [...], sino de hipótesis científicas empíricamente fundamentadas que, como tales, pueden ser el origen de ulteriores generalizaciones e hipótesis secundarias». 
pende crucialmente de la cantidad y calidad de las descripciones sincrónicas (Lehmann 1969).

La aplicación de los dos métodos se da por pasos semejantes a aquellos con los que la linguiística (estructural) sincrónica (cf. Alonso-Cortés 2002) procede para la determinación de las formas profundas a partir de las formas superficiales (cf. Hoenigswald 1965; pero considérense también las precauciones de Rankin 2003 sobre este punto). Aunque en la práctica dichos pasos no poseen una ordenación estricta, pues incluso pueden aplicarse en paralelo y permitir saltos y regresos, idealmente se distinguen los siguientes (basados en Campbell 1999 y Rankin 2003):

1. Establecimiento de las formas a comparar

2. Establecimiento de las correspondencias

\section{Reconstrucción}

4. Evaluación intra e interlinguiística de las reconstrucciones

El siguiente cuadro está basado en Campbell (1999: 115-122). Se trata de los criterios según los cuales opera la reconstrucción (paso 3). Además, en él cotejo la pertinencia de dichos criterios para el MC y el MRI.

\begin{tabular}{|l|c|c|}
\hline \multicolumn{1}{|c|}{ Reconstrucción } & MC & MRI \\
\hline $\begin{array}{l}\text { Direccionalidad: algunos cambios suelen suceder en una dirección } \\
(\mathrm{A}>\mathrm{B}) \text { pero no en la otra }(\mathrm{B}>\mathrm{A}) .\end{array}$ & SÍ & Sí \\
\hline $\begin{array}{l}\text { Área mayor: se toma como protoforma la forma que tenga mayor } \\
\text { distribución dialectal. }\end{array}$ & Sí & NO \\
\hline $\begin{array}{l}\text { Factorización: se observan los rasgos comunes a las formas com- } \\
\text { paradas y se los atribuye a la forma reconstruida. }\end{array}$ & Sí & Sí \\
\hline $\begin{array}{l}\text { Economía: cuando hay varias alternativas de reconstrucción, se } \\
\text { opta por la que requiere la menor cantidad de cambios en su } \\
\text { derivación. }\end{array}$ & SÍ & Sí \\
\hline
\end{tabular}

\section{Evaluación de los antecedentes de acuerdo con el MC y el MRI}

Los siguientes trabajos constituyen el estado de la cuestión acerca de la historia del sufijo tenencial. Realizaré la discusión de cada uno de ellos según el orden cronológico original de las publicaciones. 
3.1. Solís (1989)2 y Solís y Chacón (1989). En ambos trabajos se maneja la misma hipótesis respecto de la historia de -yuq, pero haré referencia únicamente al segundo de ellos, dado que en él la hipótesis es desarrollada con un poco más de detalle.

En Solís y Chacón (1989), obra de carácter sincrónico, los autores realizan breves digresiones acerca de la naturaleza del tenencial -yuq. Señalan que dicho sufijo sería una composición de dos morfemas, que identifican como el "verbo numérico" •yu-3, de significado equivalente a 'tener' o 'haber', y el sufijo agentivo $-q$ en función de relativizador. Añaden también que, aunque -yu- es morfológicamente un sufijo, actúa como verbo, al igual que otros tantos sufijos que antes habrían sido raíces verbales.

En primer lugar, debo destacar la incongruencia de los autores cuando hablan en términos sincrónicos tanto de un verbo •yu- como de un sufijo •-yu (cf. Cerrón-Palomino 1997). Intentando resolver dicha incongruencia, interpreto que para ellos -yuq sería una secuencia recurrente de dos sufijos, •-yu-q, donde $\bullet-y u$ - sería un sufijo que antes habría sido un verbo, y -q sería el sufijo agentivo, de modo que la historia del sufijo tenencial podría ser esquematizada así: •-yu-q < •yu-q. Luego de esto, y admitiendo el carácter digresivo de la discusión acerca del sufijo en cuestión en la obra de Solís y Chacón, puedo hacer los siguientes comentarios:

A. Este trabajo no es una reconstrucción sino una propuesta (en el sentido de Landerman 1991), pues no ha sido realizado siguiendo los pasos ni teniendo en cuenta los criterios de reconstrucción (ver 2).

B. Esta es una propuesta sobre el origen más remoto del sufijo en cuestión, pues intenta remontarse a sus constituyentes elementales.

C. En esta propuesta, los constituyentes elementales se identificarían con un verbo $\bullet$ yu- y el sufijo agentivo $-\mathrm{q}$.

D. Como propuesta, es una desde la cual no se podría derivar ni diacrónica ni sincrónicamente el sufijo en cuestión, ya que no podría dar cuenta de su morfofonología (ver 4.4.2).

E. Esta propuesta es de naturaleza impresionista. En tanto no ofrece argumentos, la identificación del último segmento de -yuq con el sufijo agentivo -q (y, a partir de ahí, el análisis de -yuq como •-yu-q y la independización de -yu-) es superficial, está basada únicamente en la semejanza fonológica, la

2 Este trabajo se halla incorporado también en Solís (1997).

3 Uso el símbolo "•” para significar que la forma es hipotética, propuesta. A menos que señale lo contrario, reservo el usual símbolo “*” para las formas reconstruidas (generadas con la metodología consolidada de la LH). 
cual no basta para concluir la identidad de dos morfemas, o partes de ellos, desde una perspectiva diacrónica.

En síntesis, el análisis de -yuq como $\bullet-y u-q$ (proveniente de $\bullet y u-q)$ no puede ser sostenido como una conclusión sino solo como una hipótesis a poner a prueba, y no en una perspectiva sincrónica sino diacrónica; y como hipótesis, es una desde la cual el sufijo en cuestión no podría derivarse ni diacrónica ni sincrónicamente.

3.2. Landerman y Weber (en Weber 2008)4. En esta obra de carácter sincrónico, Weber discute la naturaleza de -yuq a propósito del comportamiento irregular de la secuencia ni (ver 4.4.2). Para ello, refiere una comunicación personal del linguiista Peter Landerman, quien sugeriría que dicho sufijo habría sido •yyuq en una etapa previa, postulación para la cual habría evidencias dialectales. A partir de la sugerencia de Landerman, Weber interpreta que dicha forma podría ser en la actualidad la representación profunda del sufijo en cuestión, cuya realización superficial como [yoq] devendría de una reducción de /yy/ en /y/.

Dada la brevedad de la comunicación personal de Peter Landerman y la interpretación de Weber, es necesario, a su vez, que yo interprete y explicite algún punto del trabajo:

a. Con una forma profunda como •-yyuq se justificaría la aparición aparentemente inmotivada de ni en la derivación con bases nominales acabadas en consonante: ni aparecería para evitar una secuencia (profunda) triconsonántica, insilabeable en quechua (ver 4.3).

Luego de esto, y admitiendo también aquí lo conciso de la discusión abierta por Landerman, puedo hacer las siguientes observaciones:

A. Este trabajo parece tener visos de reconstrucción, pues, supuestamente, se parte de las características morfofonológicas del sufijo en cuestión y se sugiere que está basado en un cotejo dialectal. Sin embargo, ¿cuáles podrían ser esas referidas "evidencias dialectales" que autorizarían postular •-yyuq y cómo las utilizó Landerman? Entre tanto estos puntos no sean expuestos, el trabajo solo puede ser aceptado como una propuesta (en el mismo sentido de arriba).

B. A diferencia de Solís (1989) y Solís y Chacón (1989), esta propuesta no intenta remontarse al origen del sufijo en cuestión, ya que no se cuestiona acerca de una posible constitución interna diacrónica de -yuq, sino solo a una etapa inmediatamente anterior capaz de explicar su sincronía.

4 Segunda edición castellana de Weber 1989. 
C. Como propuesta, •-yyuq sí puede dar cuenta del comportamiento irregular de ni (ver 4.4.2) y de la realización fonética del tenencial como [yoq]. En otras palabras, dicha propuesta soluciona un problema y es derivable sincrónicamente.

En síntesis, •-yyuq, sea como propuesta de forma profunda y/o de una etapa inmediatamente anterior, constituye una buena hipótesis, a ser puesta prueba, para la explicación del comportamiento sincrónico del sufijo en cuestión.

3.3. Cerrón-Palomino (1997). Este trabajo está abocado directamente a la diacronía de varios sufijos quechuas, entre ellos el tenencial. Por ello, es en el que se intenta exhibir de forma más transparente y sistemática una línea argumental. Resolviendo alguna debilidad del trabajo5, puedo señalar que el autor procede de la siguiente manera:

Primero, observa que, a pesar de su gran productividad en las demás posiciones, el fonema uvular /q/ no aparece mucho en la posición de final de palabra de las raíces no verbales; que una parte del grupo de palabras donde el fonema /q/ sí aparece en dicha posición se trata de gramaticalizaciones evidentes de una construcción de raíz verbal más sufijo agentivo; y que otra parte del grupo de palabras son nombres y adjetivos que también se pueden presentar sin dicho fonema o que pueden sustituirlo. Luego, asume6 que también en este último caso se puede identificar el segmento /q/ con el sufijo-q. Después, observa los rasgos gramaticales del sufijo agentivo, el cual, sincrónicamente, solo se adhiere a bases verbales; asume que esto debió ser así también en el pasado; concluye que debió haber una base verbal entre el sufijo agentivo y dichas raíces no verbales; e hipotetiza que dicha base verbal pudo haber sido el verbo ka- 'ser'. Luego, asume que ese mismo análisis puede aplicarse a los sufijos en los que el segmento /q/ aparece en posición final. Por lo anterior, la historia de -yuq podría ser esquematizada así: -yuq $<\bullet-y u$ ka-q, donde •yu7 sería el sufijo nominal original con el significado aproximado de 'con', y toda la construcción significaría 'el que es con...'. Finalmente, a modo de prueba de que la desaparición del verbo ser es un fenómeno regular en las lenguas y especialmente en el quechua, el autor presenta instancias quechuas de lenición

5 Ver siguiente nota a pie de página.

6 El autor argüye que la reductibilidad del grupo de nombres y adjetivos a formas acabadas en vocal, sin el fonema /q/, estaría "probando, de refilón, la identificación del segmento final como una antigua marca agentiva» (Cerrón-Palomino 1997: 188). Sin embargo, dicha reductibilidad de las formas no verbales no puede probar que, en ese caso, el segmento /q/ es o fue -q, ya que la aparición de -q con formas no verbales es precisamente la hipótesis que se quiere probar. Circularmente, el autor ha tomado aquí las observaciones que han generado su hipótesis como demostraciones de su hipótesis, por lo que incurre en petición de principio.

7 ¿Un sufijo de caso? 
del artículo wanka -kaq, proveniente de *ka-q; de elipsis del verbo ka- 'ser' en perífrasis verbales en alguna variedad ancashina, como en la alternancia wiya-shqa ka-a wiya-shqa-a 'he oído'; y de lenición del verbo ser en frases atributivas de quechua ecuatoriano, como en la alternancia Juana-mi ga-ni Juana-mi a-ni $\sim$ Juana-m-a-ni; e instancias aimaras de lenición del verbo ser.

Luego de esto, puedo hacer las siguientes observaciones:

A. Este trabajo tiene aspectos de reconstrucción, como es la base empírica de reconocer y factorizar las características fonotácticas del fonema /q/ y las características morfosintácticas del sufijo -q. Sin embargo, no reconoce y, por tanto, no factoriza las características morfofonológicas ni los correlatos dialectales de -yuq, el sufijo a cuya historia se aboca. Por ello, es más una reconstrucción del sufijo -q que del sufijo -yuq. En cuanto al sufijo -yuq, entonces, este trabajo es una propuesta.

B. Esta propuesta intenta remontarse hasta los componentes originales del sufijo en cuestión. Según ella, la historia de -yuq podría esquematizarse de la siguiente manera: -yuq $<\bullet-$ yu ka-q.

C. Como propuesta, es una desde la cual no se podría derivar ni diacrónica ni sincrónicamente el sufijo en cuestión, pues, como quedó señalado arriba, no considera ni sus características morfofonológicas propias ni sus correlatos dialectales.

D. La desaparición del verbo ka- 'ser' no parece un fenómeno tan regular en el quechua como el autor propone. Por ejemplo, en el caso de la lenición del artículo -kaq del dialecto wanka, se trata no de la desaparición del verbo sino de un sufijo particular proveniente de una construcción con él; es decir, se está ante la lenición del sufijo -kaq, no del verbo ka-; aun más, en alguna variedad huanuqueña que, sea por préstamo o por desarrollo independiente, también posee el artículo, dicha lenición no ha sucedido (cf. Weber 2008); por tanto, la elisión del sufijo -kaq es un desarrollo tardío muy particular del wanka. Igualmente, en el caso de la elipsis en cierta variedad ancashina y de la lenición en el ecuatoriano, se observa que ellas suceden ante los morfemas de persona y no ante otros morfemas.

E. La lenición del verbo kanka- en aimara no viene al caso. Intentar probar mediante el análisis del aimara las consecuencias de una hipótesis sobre el quechua simplemente no es conducente, a pesar del gran parecido entre ambas lenguas. Los datos aimaras pueden servir como la motivación, pero de ningún modo como la prueba de una hipótesis sobre el quechua. 
En síntesis, -yuq < $<-y u$ ka-q es una hipótesis cuyas consecuencias se observan de forma irregular8 y desde la cual el sufijo en cuestión no podría derivarse ni diacrónica ni sincrónicamente.

3.4. Calvo Pérez (2006). En este trabajo, el autor realiza una digresión según la cual el sufijo -yuq sería «derivado del interiorativo -y[k]u más el agentivo -q» (Calvo Pérez 2006: 158, nota 1). Dada la extrema brevedad de la digresión, resulta necesario que la interprete para poder hallarle sentido:

a. El autor parece sugerir que el sufijo tenencial -yuq se habría derivado de los sufijos -yku y-q, y que, antes o después del reanálisis de ambos sufijos en uno solo, en -yku habría obrado un debilitamiento del segmento k. En efecto, debo señalar que la alternancia -yku -yu del "interiorativo" se registra en dialectos cuzqueños (cf. Cusihuamán 2001, Cerrón-Palomino 2008)9.

Luego de esto, y admitiendo aquí también lo conciso de la digresión de Calvo Pérez, puedo hacer las siguientes observaciones:

A. Este trabajo no es una reconstrucción sino una propuesta (ver 2).

B. Esta es una propuesta sobre el origen más remoto del sufijo en cuestión, pues intenta remontarse a sus constituyentes elementales.

C. Según esta propuesta, los constituyentes elementales del tenencial serían el sufijo verbal -yku y el derivador nominal deverbativo -q, el ya mencionado "agentivo".

D. Como propuesta, es una que resulta inderivable diacrónicamente, porque no se conoce un proceso de elisión del fonema $\mathrm{k}$ en esa posición -ni en ninguna otra- atribuible a la protolengua y, sobre todo, porque -yku es, diacrónica y sincrónicamente, un sufijo verbal, incompatible con bases nominales, las cuales son el tipo de base a las que se agrega el tenencial10.

8 Por supuesto, esta objeción se aplica a cualquier hipótesis acerca de la formación del sufijo en cuestión. Luego de extraídas todas las conclusiones reconstructivas posibles a partir de la evolución conocida del protoquechua, las historias de las raíces y sufijos que no caen en ese ámbito, el de los cambios regulares, no pueden más que ser trabajadas apelando a información parcial, a cambios irregulares y demás.

9 En una obra panorámica sobre el quechua, Adelaar y Muysken sostienen que -yku «alternates with -yu- [...] in many dialects» (2004: 202); sin embargo, no parece haber datos que respalden esta afirmación. Lo que sí se registra abundantemente es la alternancia -yka $\sim$-ya del durativo de los quechuas no sureños, un sufijo completamente diferente. Según se verá más delante, el sufijo tenencial -yuq tiene esta forma -en lo que respecta al segmento y- por lo menos desde la protolengua.

10 Para que esta propuesta fuese coherente, habría que proponer un verbo entre la base y el sufijo -yku, de manera semejante a como hace Cerrón-Palomino (supra), o proponer protorrasgos diferentes para ${ }^{*}$-yku. 
Paradójicamente, sin embargo, observando solo la información segmental e ignorando las incompatibilidades morfosintácticas, la propuesta sí resultaría derivable sincrónicamente11.

En síntesis, la propuesta de -yuq $<\bullet-y k u-q$ es una propuesta irregular que, aunque en cierto sentido resulta derivable sincrónicamente, muestra incoherencias morfosintácticas, tanto diacrónicas como sincrónicas.

3.5. Evaluación global del estado de la cuestión. Conforme ha podido observarse, hay dos afirmaciones globales posibles acerca de los trabajos evaluados. La primera es que, aunque algunos de ellos presentan ciertos aspectos de reconstrucción, ninguno cumple cabalmente con los requisitos de los dos métodos consolidados para reconstruir lenguas (el MC y el MRI) y, en particular, el sufijo en cuestión. La segunda es que en casi todos hay una constante: la identificación del último segmento de -yuq con el sufijo -q 'agentivo', si bien solo en uno -en Cerrón-Palomino (1997)- se entrega un argumento, de tipo fonotáctico, en favor de dicha identificación.

\section{Premisas quechuas para la reconstrucción}

En esta sección expongo los datos de gramática quechua pertinentes para cualquier reconstrucción morfológica y para la del sufijo tenencial en particular.

\subsection{La sílaba quechua}

Salvo ciertos desarrollos independientes, los tipos de sílabas quechuas conservadoras son V, VC, CV y CVC, de las cuales V y VC solo son posibles si son la sílaba inicial de palabra (Cerrón-Palomino 2003: 256-258)12 y donde la C en coda vale tanto por las semiconsonantes como por el alargamiento vocálico en los quechuas que lo presentan13 (cf. Weber y Landerman 2008). Dada su generalidad, dicha tipología silábica y su distribución son atribuibles al protoquechua sin ningún problema.

La reproducción de los tipos silábicos y su distribución arrojan el esquema \#[(C)V(C)].[CV(C)]n\# (cf. Floyd 2008) para toda palabra quechua, cualquiera sea su estructura morfológica. Para los intereses de este trabajo, a partir de

11 Resultaría así si se supone que -yuq tendría una consonante subyacente, el residuo de la k de -yku (o sea, una forma como •-yCuq) y que esta secuencia subyacente de dos consonantes sería la razón de la inserción de ni (ver sección 4.4.2).

12 cf., por ejemplo, Floyd 2008, Chaparro 1985, Shaver y Shaver 2008, respecto de lo silábico excepcional; y Cerrón-Palomino 1976, Coombs y otros 1976, Cusihuamán 1976, Parker 1976, Quesada 1976, Soto 1976a, etc., respecto de lo convencional

13 Esta afirmación es válida aquí y en adelante. 
lo anterior es posible realizar las siguientes afirmaciones (algo redundantes) sobre el (proto)quechua: que en él no se daban secuencias de más de dos consonantes, que las secuencias de dos consonantes solo podían darse en interior de palabra, y que, cuando se daban, estas secuencias eran necesariamente heterosilábicas.

\subsection{Morfología quechua}

La familia quechua muestra una morfología esencialmente aglutinante y, salvo algún desarrollo muy particular, exclusivamente sufijadora (Adelaar y Muysken 2004). Una palabra quechua está formada necesariamente por una raíz con o sin sufijos, dependiendo del tipo de raíz del que se trate, donde los sufijos pueden clasificarse como derivativos, flexivos e independientes de acuerdo con una posición fija en la estructura de la palabra y con el tipo de relaciones que establecen (Cerrón-Palomino 2003). El siguiente cuadro, basado en Cerrón-Palomino (2003), muestra este orden lineal de los elementos de la palabra:

\begin{tabular}{|l|l|l|l|}
\hline \multirow{2}{*}{ Raíz } & \multicolumn{3}{|c|}{ Sufijos } \\
\cline { 2 - 4 } & Derivativos & Flexivos & Independientes \\
\hline
\end{tabular}

Finalmente, cabe destacar que dicho orden lineal, válido tanto para estructuras nominales como verbales, rige para toda la familia y es, por tanto, atribuible al protoquechua.

\subsection{Morfofonología quechua: el uso de ni}

En quechua, se recurre a la inserción de ni (cf. Weber y Landerman 2008) para, básicamente, evitar secuencias de segmentos que transgredirían la tipología silábica nativa (ver 4.1)14. Así, a partir de qunqur 'rodilla' y -n '3p. poseedora' se obtiene no *qunqurn15 sino qunqurnin para expresar 'su rodilla'. Las condiciones para esta inserción motivada de ni son morfofonológicas: se dan cuando a una base acabada en consonante se le aplica un sufijo de estructura canónica $\mathrm{C}(\mathrm{CV}(\mathrm{C}))$ (Cerrón-Palomino 2010 y cf. Floyd 2008) o, dicho de otra manera, cuando a tal base se le aplica un sufijo de forma (profunda) $\mathrm{C}$ o que comience con dos C (o sea, CC...). Ya que en la tipología estructural tradicional de los sufijos quechuas no hay sufijos que comiencen con vocal16 ni sufijos que comiencen con más de dos consonantes, entonces, toda base acabada en

14 cf. Cerrón-Palomino 1976, Coombs, Coombs y Weber 1976, Cusihuamán 1976, etc.

15 Solo aquí, el símbolo “*” representa agramaticalidad.

16 Excepciones son, por ejemplo, los sufijos prestados del castellano (cf. Quesada 1976) y ciertos desarrollos particulares (cf. Adelaar y Musken 2004, Parker 2013). 
consonante a la que se le aplicara un sufijo de estructura canónica, seguido o no de otros sufijos (en final de palabra, en este último caso), produciría secuencias o de tres $\mathrm{C}$ en interior de palabra o de dos $\mathrm{C}$ a final de palabra; secuencias anómalas, insilabeables, como ya indiqué (ver 4.1).

Por último, a un lado de la inserción motivada de ni, se registra también su uso de modo aparentemente inmotivado precisamente con el tenencial. Desarrollaré este punto en la sección 4.4.2.

\subsection{El sufijo tenencial (-yuq)}

En esta sección presento los datos pertinentes para la reconstrucción del tenencial.

\subsubsection{Caracterización}

Según Torero (2002), este sufijo está presente en todo el universo quechua y es en él uno de los más productivos 17 . Se caracteriza morfológicamente por ser un derivador nominal denominativo; es decir, se aplica a bases nominales para generar nuevos elementos nominales. El derivado alude al poseedor (sobre todo permanente) de lo referido por la base a la que se sufija. Por ello, regularmente es etiquetado como "tenencial" o "posesivo". La idea de posesión o tenencia que conlleva -yuq es muy extensa (Weber 2008) y rebasa la variedad de usos de tener y poseer en castellano. Ejemplos18:

(1) en el extinto quechua general costeño (ST19, [1560] 1995: 175)

ancha imayoc [...]. Caycóna conan angeles xutiocmi

ancha ima-yuq [...] kay-kuna kunan ángeles shuti-yuq-mi

mucho algo-TEN este-PLU ahora ángeles nombre-TEN-EVD

'muy ricos [...]. Estos agora ángeles se llaman'

(2) en el quechua general sureño (TCL20, [1584-1585] 1984: fol. 26)

rurafcanmi, huañuc ucuyoc, viñay cauçac animayoc

rura-shqa-n-mi wañu-q uku-yuq wiñay kawsa-q anima-yuq

17 cf. Adelaar 1987, Cerrón-Palomino 1976, Coombs, Coombs y Weber 1976, Cusihuamán 2001, Escribens y Proulx 1970, Parker 1965 y 1976, Quesada 1976 y 2006, Solá 1967, Soto 1976a, Weber 2008.

18 En los ejemplos (1), (2) y (4), los análisis morfológicos son míos. En el ejemplo (3), que no cuenta con versión grafémica pura en el original, he estandarizado los grafemas y las etiquetas morfológicas. Aquí y en adelante, mis análisis morfológicos no pretenden ser etimológicos, sino solo lo suficientemente aproximados.

19 Santo Tomás, Fray Domingo de

20 Tercer Concilio Limense 
hacer-PRF-3-EVD morir-AGN cuerpo-TEN siempre vivir-AGN alma-TEN 'criatura compuesta de cuerpo que muere, y de alma que nunca ha de morir'

(3) en quechua de Pachitea (Huánuco) (Weber (ed.) 1987: 115)

wamra-kuna fastidiya-ra-n "Trupa-yoq" ni-r

niño-PLU fastidiar-PSD-3 cola-TEN decir-SUB

'los niños le fastidiaban diciendo: "iRabudo!"

(4) en quechua de Pacaraos (Adelaar 1987: 64)

uchukchanay wawiyuq kaykan

uchuk-cha-nay wawi-yuq ka-yka-n

pequeño-??? hijo-TEN ser-PRG-3

'tienen crías chiquitas'

Por estar presente en todo el universo quechua, el tenencial es atribuible sin ningún problema al protoquechua.

\subsubsection{Morfofonología de la derivación con-yuq}

Según quedó señalado en la sección 4.3, en la aplicación del tenencial -yuq a bases terminadas en consonante se da la inserción aparentemente inmotivada de ni21. Ejemplos22:

(5) en la formación de numerales (panquechua)

trunka hukniyuq, trunka pusaqniyuq, trunka isqunniyuq

trunka huk-ni-yuq trunka pusaq-ni-yuq trunka isqun-ni-yuq

diez uno-0-TEN diez ocho-0-TEN diez nueve-0-TEN

'once, dieciocho, diecinueve'

(6) en quechua del Huallaga (Huánuco) (Weber 2008: 82)

eskirbaanu llapan fistakunawan munayniyuq

eskirbaanu llapan fista-kuna-wan muna-y-ni-yuq

escribano todo fiesta-PLU-COM querer-INF-0-TEN

'el escribano tiene toda la fiesta bajo su control'

21 En la literatura quechua existen algunos casos de ausencia de inserción de ni en este contexto. Los criterios de la LH (ver 2) indican que dicho comportamiento no debe ser reconstruido, sino tomado como una innovación. Se trataría, simplemente, de un proceso de regularización.

22 En el ejemplo (6), he estandarizado la versión grafémica y las etiquetas morfológicas. En el (7), el análisis morfológico es mío. 
(7) en una variedad juninense (Cerrón-Palomino 1976: 154)

lintis-ni-yuq-mi ka-ñaq wamla-kaq

lentes-0-TEN-EVD ser-PSD muchacha-ART

'la muchacha tenía lentes'

Ese uso de ni sería inmotivado porque las secuencias heterosilábicas ky, qy, ny y demás son válidas en quechua, como se observa en las raíces pukyu 'puquio' (panquechua), laqya- 'asestar un golpe...' (Soto 1976b), tsunyay 'silencio’ (Chávez y Parker 1976), etcétera. De acuerdo con Cerrón-Palomino (2010), la inserción de ni en los ejemplos responde a consideraciones eufónicas y de tradición. En efecto, atribuirle carácter tradicional a dicho comportamiento excepcional de ni ante -yuq es una de las dos respuestas posibles23. Sin embargo, en cuanto a la supuesta razón eufónica aducida por el autor, debo acusar que esta no obra cuando otro sufijo de estructura aparentemente idéntica a -yuq se aplica a bases acabadas en consonante. Se trata del sufijo limitativo -yaq, presente en quechuas centrales. Ejemplos24:

(8) en una variedad ancashina (Parker 1976: 85)

shuyaamanraqku tsayyaqqa

shuya-:-man-raqku tsay-yaq-qa

esperar-1-CND-NEG.ENF eso-LIM-TOP

'no debo esperar hasta eso'

(9) en el quechua del Huallaga (Huánuco) (Weber 2008: 275)

chaykuna uchkun rurikama pampananpaq alli kananyaq

chay-kuna uchku-n ruri-kama pampa-na-n-paq alli ka-na-n-yaq

ese-PLU cavar-3 interior-LIM enterrar-SUB-3-PRO bueno ser-SUB-3-LIM

'cavan hacia abajo [...] hasta que es suficientemente profundo para enterrarlo'

Así, se observa que atribuirle a la inserción de ni ante -yuq una motivación eufónica resulta ser un recurso adhoc. Entre tanto, dicho comportamiento irregular, que a partir de ahora puede ser llamado "la anomalía morfofonológica", espera una mejor respuesta (ver 5.2).

23 Coseriu (1978: 123): «todo aquello que en las gramáticas corrientes se halla señalado como "otra posibilidad" o como "excepción", es un reflejo de lo diacrónico en lo sincrónico, ya sea como constitución de un modo nuevo o como persistencia de un modo antiguo».

24 En el ejemplo (8), el análisis morfológico es mío. En el (9), solo he estandarizado la versión grafémica. 
Dado que se halla en todo el universo quechua, puede afirmarse que la anomalía morfofonológica existe por lo menos desde la etapa protoquechua.

\subsubsection{Las formas ferreñafanas}

Si bien el tenencial se registra como -yuq en la gran mayoría de los quechuas, no es esa la única forma en la que se presenta. La consideración de tantos cognados como sea posible es justamente una de las exigencias para la reconstrucción de la que los antecedentes de este trabajo adolecen. Esas otras formas son las variantes ferreñafanas -yqu (Shaver y Shaver 2008) e -yqun (cf. Taylor 1994 y Weber (ed.) 1987)25, ambas 'tenencial'. Aparte de la glosa, la relación de cognados entre los sufijos -yuq e -yqu(n) se hace evidente al simplemente observar que entre ellos media algo más que una metátesis. Determinar cuál de ellas es la forma innovadora (o sea, la metatética) y, por tanto, cuál la conservadora, es una de las etapas de la reconstrucción (ver 5.1).

\section{Reconstrucción del sufijo -yuq}

En esta sección realizo la reconstrucción del tenencial. Para una exposición más clara, en todo momento intento mantener deslindados los pasos reconstructivos, a pesar de lo dicho en la sección 2 acerca de su aplicación en paralelo.

\subsection{Aplicación del MC}

Establecimiento de las formas a comparar. Los cognados son -yuq (en la inmensa mayoría de quechuas), -yqu (Incahuasi) e -yqun (Ayamachay).

Establecimiento de las correspondencias. Entre los cognados se observa, en primer lugar, una correspondencia perfecta a través de la metátesis: $-\mathrm{y} 1 \mathrm{u} 2 \mathrm{q} 3$ $\sim-\mathrm{y} 1 \mathrm{q} 3 \mathrm{u} 2 \sim-\mathrm{y} 1 \mathrm{q} 3 \mathrm{u} 2(\mathrm{n}) ; \mathrm{y}$, en segundo lugar, una correspondencia $\emptyset \sim \emptyset \sim \mathrm{n}$.

Evaluación intra e interlinguiística. Una revisión de los trabajos en los que se registran las formas ferreñafanas (ver 4.4.3.) indica que estos quechuas son particularmente proclives a la metátesis. Así, sufijos protoquechuas como *paq, ${ }^{*}$-pis (Parker 2013) y ${ }^{*}$-nčik se presentan en quechuas ferreñafanos como -pqa, -psi y -nčki, respectivamente (Shaver y Shaver 2008). Por lo anterior, los cognados quedan reducidos (o sea, restablecidos: paso 1) a -yuq (en la inmensa mayoría de quechuas) y la forma reconstruida *-yuqn (protoayamachano).

Reconstrucción. Por el criterio de direccionalidad, se debería reconstruir el segmento nasal presente en *-yuqn, ya que la elisión de cualquier segmento es más común que su epéntesis. Por el criterio de área mayor, se debería recons-

25 Taylor escribe -yxun. 
truir una forma idéntica a -yuq, ya que la situación según la cual únicamente el quechua de Ayamachay hubiese añadido la nasal es más probable que la situación según la cual todos los demás quechuas, ya independizados, la hubiesen elidido. La factorización se aplica vacuamente, porque los tres segmentos que los cognados tienen en común son idénticos. Por el criterio de economía, los cognados quedan igualados, pues cualquiera de ellos pudo haber originado al otro con la aplicación de una sola regla (inserción de nasal en el sentido -yuq $>$-yuqn o elisión de nasal en el sentido -yuqn $>-$ yuq). De este modo, por ahora, el sufijo tenencial puede ser reconstruido como ${ }^{*}$-yuq(n).

Por último, una evaluación intralinguiística del resultado señala que este no es viable, dado que el patrón que podían tener los sufijos en la etapa protoquechua, que se desprende del respectivo patrón silábico, es el siguiente: $-\mathrm{C}(\mathrm{CV}(\mathrm{C}))$; en específico, no podía haber una secuencia de consonantes en el límite derecho. Por ello, sostengo la reconstrucción *-yuq(n) solo tentativamente y la someto a revisión en la siguiente subsección.

\subsection{Aplicación del MRI}

Puesto que el MRI aprovecha las irregularidades morfológicas, en este punto resulta pertinente invocar la anomalía morfofonológica, según la cual ni aparece de forma aparentemente inmotivada ante el tenencial. Así, sugiero que el tenencial puede ser reconstruido a partir del efecto que provoca en ni; es decir, como un sufijo que empieza con dos consonantes, donde la nueva $\mathrm{C}$ podría colocarse antes o después del segmento yod: *-Cyuq o *-yCuq. Ahora, si se recuerda que los dialectos ferreñafanos son proclives a la metátesis (evaluación interlinguiística), es posible identificar aquella nueva $C$ de la reconstrucción interna con la nasal reconstruida comparativamente, de lo que resultan dos opciones posibles: ${ }^{*}-$ nyuq o ${ }^{*}-$ ynuq. De este modo, la consonante nasal que el MC invita a reponer en el límite derecho pero que, de acuerdo con la tipología silábica y sufijal del protoquechua, no puede colocarse ahí, resulta ser la consonante extra que el MRI exige reponer en el límite izquierdo. Esta nasal habría sido removida de su posición original, mediante metátesis, en el transcurso del protoayamachano al ayamachano moderno, en tanto que habría desaparecido completa o casi completamente en la protolengua predecesora de las demás ramas, dejando solo su efecto morfofonológico: la inserción de ni.

\section{Conclusiones respecto de la reconstrucción}

Acerca de la reconstrucción del tenencial que he llevado a cabo en este trabajo, puedo concluir lo siguiente: 
A. Tiene sustento empírico.

Esta es una característica definitoria de mi reconstrucción, puesto que he seguido los requisitos del MC y del $\mathrm{MH}$, los cuales son instrumentos empíricos.

B. Es derivable sincrónicamente.

Para la derivación sincrónica del tenencial, bastaría proponer, por ejemplo, que su representación abstracta tendría una consonante subespecificada e irrealizable (por ejemplo, /-Cyuq/ $\rightarrow[-$ yuq $]$ ), pero justificada en tanto residuo de una antigua consonante (/-nyuq/ > /-Cyuq/, siguiendo con el ejemplo).

C. Presenta un problema de derivación diacrónica.

El problema diacrónico que presenta es que no se conoce un cambio fonológico regular que, del protoquechua a los quechuas actuales, haya eliminado nasales. Sin embargo, no puede ponerse en duda la existencia de uno o varios procesos irregulares con las mismas consecuencias. Por ejemplo, Domingo de Santo Tomás ofrece una evidencia en el extinto quechua costeño: la entrada turomaya para una palabra que figura como turumanya tanto en los vocabularios antiguos como en los actuales (cf. Szemiñski 2006). Asimismo, Torero (1968) y Parker (2013) acusan la elisión de nasales en coda en lexemas del quechua de Huaylas, como puede observarse en los cognados qa:yan y qanyan, ambos 'ayer', o en qanya:tin 'antes de ayer', que remite a una derivación con el sufijo * ${ }^{-n t i(n) . ~ P o r ~ u ́ l t i m o, ~ L a n d e r m a n ~(1991) ~ s e n ̃ a l a ~ l a ~}$ caída sincrónica, morfofonológica, de la nasal bilabial del sufijo -mu en un dialecto wanka. De este modo, para el caso del tenencial, solo es necesario suponer un cambio de naturaleza semejante en una etapa temprana26.

D. Resuelve un problema afín: la anomalía morfofonológica.

La reconstrucción del tenencial que he realizado en este trabajo explica también la anomalía morfofonológica (ver 4.4.2): la secuencia ni no tendría un comportamiento inusual ante el tenencial, sino absolutamente regular, puesto que el tenencial sería o habría sido un sufijo con dos consonantes iniciales y por ello incitaría la inserción de ni.

26 Este análisis podría también dar cuenta de la protoforma del sufijo privativo, la cual sería *-nnaq y se presentaría en la actualidad, además de como -nnaq (Cerrón-Palomino 1976, Parker 1976), en las formas -:naq (Parker ídem) y -naq (Cusihuamán 2001, Soto 1976a) como producto de la pérdida de la primera nasal; y en la forma -ynaq (Weber 2008) acaso como una reinterpretación posterior, a partir de la inserción de la secuencia ni: /ni+:naq/ > /ni+ynaq/. 


\section{Nuevas hipótesis}

Para terminar este trabajo, me referiré, de un modo menos estricto, a las nuevas hipótesis que surgen a partir de *-nyuq, una de las opciones de reconstrucción del tenencial. Defiendo esta forma porque, por una parte, la existencia de un proceso histórico irregular de elisión de nasales se verifica más en coda que en ataque (situación que se requeriría para argumentar en pro de *-ynuq); $y$, por otra, porque encaja en un formato de sufijos nominales, especialmente derivativos, con consecuencias realmente interesantes.

Derivativos

-nti(n) (panquechua)

-nnin (Weber 2008)

-nnaq (Parker 1976)

-mpa (Cusihuamán 2001)

-nka (Cusihuamán 2001, Soto 1976a)
Flexivo

-nta (Soto1976a)

Según se observa, todos esos sufijos nominales de significados diferentes coinciden en la consonante inicial, situación que difícilmente puede ser producto de la casualidad. Más bien, si se recuerda que los derivadores nominales crean nombres, palabras que hacen, per se, referencia a la tercera persona, y se sabe que el sufijo de tercera persona (poseedora), $-n$, tiene dicha forma desde por lo menos la etapa protoquechua (Parker 2013), es posible, entonces, identificar ese segmento inicial de los derivadores con el sufijo -n de tercera persona. Así, sugiero que no habría aquí casualidad sino sistematicidad; es decir, que dichos sufijos serían coincidentes ahora porque habrían tenido historias con algún punto en común: todos habrían incorporado ese sufijo -n flexivo, lo habrían reanalizado como parte integrante. Ahora bien, dado que, por lo menos desde el protoquechua, los sufijos derivativos preceden a los flexivos (ver 4.2.), se sigue que antes de la formación de *-nyuq habría existido un límite de palabra entre $-\mathrm{n}$ y -yuq: es la única manera coherente en que un sufijo derivativo podría haberse constituido teniendo como primer segmento, a la izquierda, un elemento originalmente flexivo27. De este modo, ${ }^{*}$-nyuq permitiría retroceder hasta un hipotético $\bullet-$ n yuq.

Finalmente, me gustaría dar un paso más y, sobre la base de la argumentación fonotáctica de Cerrón-Palomino (1995; ver 2 aquí) y las referidas consecuencias de *nyuq, sugerir que, a su vez, $\bullet$-n yuq provendría de una construcción de relativo con el sufijo -q 'agentivo' aplicado a una raíz verbal transitiva

27 Esta misma lógica se sigue para cada uno de los sufijos derivativos arriba enlistados: entre la nasal inicial y el resto de segmentos habría mediado un límite de palabra. 
-yu- 'tener' cuyo OD estaría marcado con el sufijo -n de tercera persona: • -n yu-q28.

\section{Referencias bibliográficas}

ADELAAR, Willem (1987). Morfología del quechua de Pacaraos. Lima: UNMSM.

ADELAAR, Willem y Pieter MUYSKEN (2004). The Languages of the Andes. Cambridge: Cambridge University Press.

ALONSO-CORTÉS, Ángel (2002). Linguíística. Madrid: Cátedra.

CALVO PÉREZ, Julio (2006). "La oración de relativo en quechua: la aportación de los misioneros lingüistas" en Verba Hispánica, XIV; pp. 155-172.

CAMPBELL, Lyle (1999). Historical Linguistics: An Introduction. Massachusetts: MIT Press.

CERRÓN-PALOMINO, Rodolfo (1976). Gramática quechua Junín-Huanca. Lima: IEP/MINEDU.

CERRÓN-PALOMINO, Rodolfo (1997). "Reducción y ensamblaje en la formación de sufijos del quechua” en Lexis. XXI, 2; pp. 185-210

CERRÓN-PALOMINO, Rodolfo (2003). Linguística quechua. Cusco: C.E.R.A. "Bartolomé de las Casas".

CERRÓN-PALOMINO, Rodolfo (2008). Quechumara. Estructuras paralelas del quechua y del aimara. La Paz: Plural editores. La primera edición es de 1993.

CERRÓN-PALOMINO, Rodolfo (2010). "Sobre el morfo vacío -ni del quechua” en Carlin, Eithne y Simon van de Kerke (eds.), Linguistics and Archaeology in the Andes. Leiden/Boston: Brill. pp. 177-189.

COOMBS, David y otros (1976). Gramática quechua San Martín. Lima: IEP/MINEDU.

COSERIU, Eugenio (1981). Lecciones de linguística general. Madrid: Gredos.

CUSIHUAMÁN, Antonio (2001). Gramática quechua Cuzco-Collao. Cusco: C.E.R.A. "Bartolomé de las Casas".

CHAPARRO, Carmelo (1985). Fonología y lexicón del quechua de Chachapoyas. Lima: Editorial Sagsa.

CHÁVEZ, Amancio y Gary PARKER (1976). Diccionario quechua Áncash-Huailas. Lima: IEP/MINEDU.

ESCRIBENS, Augusto y Paul PROULX (1970). Gramática del quechua de Huaylas. Lima: UNMSM.

FLOYD, Rick (2008). "Canonicidad CV, pérdida de /q/ y cambio de lenguaje en el wanka" en Parker, Stephen (ed.), Estudios Etnolinguísticos II. Lima: ILV. pp. 62-80. La primera edición es de 1992.

28 La evaluación de las consecuencias de esta hipótesis constituye la segunda y última parte del proyecto mayor aludido al inicio del presente trabajo. Para dicha evaluación, remito a Faucet (en preparación). 
HOENIGSWALD, Henry (1965). Language Change and Linguistic Reconstruction. Chicago/Londres: The University of Chicago Press.

LANDERMAN, Peter (1991). Quechua dialects and their classification. Tesis doctoral, University of California.

LANDERMAN, Peter y David WEBER (2008). "La interpretación de las vocales largas en el quechua” en Coombs, Heidi y Agot Bergli (eds.), Estudios quechuas II. Lima: ILV.; pp. 12-28.

MORENO CABRERA, Juan Carlos (1997). "Sobre algunos problemas actuales de la Linguiística Histórico-Comparativa” en Revista española de Linguiística, 27; pp. 77-105.

PARKER, Gary (1963). “La clasificación genética de los dialectos quechuas”. Revista del Museo Nacional, XXXII, pp. 241-252.

PARKER, Gary (1965). Gramática del quechua ayacuchano. Lima: UNMSM.

PARKER, Gary (1976). Gramática quechua Áncash-Huailas. Lima: IEP/MINEDU.

PARKER, Gary (2013). Trabajos de Linguiística histórica quechua. Lima: PUCP.

QUESADA, Félix (1976). Gramática quechua Cajamarca-Cañaris. Lima: IEP/MINEDU.

QUESADA, Félix (2006). Quechua de Cajamarca. Morfología. Fonología. Sintaxis. Lima: Editorial Mantaro.

RANKIN, Robert (2003). "The Comparative Method" en Joseph, Brian y Richard Janda (eds.), The Handbook of Historical Linguistics. Massachusetts: Blackwell. pp. 183-212.

SANTO TOMÁS, Fray Domingo de (1995) [1560]Grammatica o arte de la lengua general de los Indios de los Reynos del Perú. Cuzco: C.E.R.A. CBC.

SHAVER, Dwight y Gwynne SHAVER (2008). "Un bosquejo de la metátesis en el quechua de Incahuasi” en Parker, Stephen (ed.), Parker, Stephen (ed.), Estudios Etnolinguiísticos I. Lima: ILV. pp. 5-14. La primera edición es de 1989.

SOLÁ, Donald (1967). Gramática del quechua de Huánuco. Lima: UNMSM.

SOLÍS, Gustavo (1989). Sistemas de denominación toponímica quechua y aru y racionalidad extralinguiística en toponomástica. Lima: CILA-UNMSM.

SOLÍS, Gustavo (1997). La gente pasa, los nombres quedan. Introducción en la Toponimia. Lima: Lengua y Sociedad.

SOLÍS, Gustavo y Jorge CHACÓN (1989). Linguiística y Gramática Runa-simi chanka. Lima: UNESCO-AGFUND/MINEDU.

SOTO, Clodoaldo (1976a). Gramática quechua Ayacucho-Chanca. Lima: IEP/MINEDU.

SOTO, Clodoaldo (1976b). Diccionario quechua Ayacucho-Chanca. Lima: IEP/MINEDU.

SZEMIÑSKI, Jan (2006). Léxico quechua de Fray Domingo de Santo Tomás. Lima: Ediciones El Santo Oficio.

TAYLOR, Gerald (1994). Estudios de dialectología quechua. Lima: EUNE.

TERCER CONCILIO LIMENSE (1984) [1584-1585]. Doctrina Christiana, y catecismo para instrvccion de los Indios... Edición facsimilar. Lima: Ediciones Copé. 
TORERO, Alfredo (1964). "Los dialectos quechuas". Anales Científicos de la Universidad Agraria. 2, pp. 446-478.

TORERO, Alfredo (1968). "Procedencia geográfica de los dialectos quechuas de Ferreñafe y Cajamarca". Anales Científicos de la Universidad Agraria. Lima, 3-4, 291-316.

TORERO, Alfredo (2002). Idiomas de los Andes. Linguística e Historia. Lima: IFEA/Editorial Horizonte.

WEBER, David (2008). Una gramática del quechua del Huallaga (Huánuco). Lima: ILV.

WEBER, David (ed.) (1987). Juan del Oso. Pucallpa: ILV.

\section{Apéndice: Tabla de abreviaturas y símbolos}

1 primera persona

2 segunda persona

3 tercera persona

0 sin valor morfológico

AGN agentivo

ART artículo

CND condicional

COM comitativo

EVD evidencial

INF infinitivo

LIM limitativo

NEG.ENF negación enfática

PLU plural

PRF perfectivo

PRG progresivo

PRO propósito

PSD pasado

SUB subordinador

TEN tenencial

TOP topicalizador

$\rightarrow \quad$ se realiza sincrónicamente como

$>\quad$ deriva diacrónicamente en

$<\quad$ deriva diacrónicamente de 